\title{
Uma sessão de exercícios de treino de força não promove alterações negativas na estrutura venosa dos membros inferiores em homens
}

\author{
One session of strength exercise does not promote negative changes in \\ the structure venous leg and thigh in men
}

\author{
Adenilson de Araújo Júnior, ${ }^{1}$ Ravi Targino Araújo, ${ }^{2}$ Rodrigo Ramalho Aniceto, ${ }^{3}$ Leonardo \\ Santos Oliveira, ${ }^{4}$ Jefferson Novaes, ${ }^{5}$ Maria Socorro Cirilo Sousa, ${ }^{3}$ José Vilaça-Alves ${ }^{6 *}$

O aumento do diâmetro venoso está associado à incompetência do sistema nos membros inferiores. O objetivo deste estudo foi analisar o efeito de uma sessão de exercícios de treino de força (TF) sobre o diâmetro venoso superficial (DV) dos membros inferiores (MI). Vinte e dois homens (22 \pm 2 anos, $81.2 \pm 13.4 \mathrm{~kg}, 173.0 \pm 4.0 \mathrm{~cm}$ e $18.6 \pm 3.3 \%$ de percentagem de gordura estimada), realizaram um circuito com agachamento, avanço e flexão plantar, sendo 3 séries de 6 repetições (3 minutos de intervalo) com carga de $85 \%$ (1-RM). Os DVs da veia safena interna (VSI), dos MI, foram medidos em repouso e imediatamente após cada série, com eco-Doppler colorido. Realizou-se um teste de Anova one-way para medidas repetidas. Para as VSI, de ambos os MI, observou-se uma diminuição dos DVs, ao longo das séries. Ademais, entre as séries, houve uma queda significativa entre a primeira e segunda $(p=0.009)$ e um aumento entre a segunda e terceira séries $(p=0.027)$ para a VSI direita, e para a VSI esquerda, apenas as medidas após a primeira e segunda foram diferentes $(p=0.001)$. Houve uma tendência de redução do DV após uma sessão aguda de exercício de força. Conclui-se que a prática de uma sessão de exercícios de TF não promove alterações negativas na estrutura das veias de homens treinados.

Palavras-chave: veias varicosas, adultos, extremidade inferior, exercício de força, performance atlética

ABSTRACT

The increase of the venous diameter is associated with the incompetence of the venous system of lower limb. In order to analyze the acute effects of one session of strength exercise (SE) on superficial venous diameter (VD) of the lower limbs (LL). Twenty-two men $(22 \pm 2$ years old, $81.2 \pm 13.4 \mathrm{~kg}$ weight, $173.0 \pm 4.0 \mathrm{~cm}$ height and $18.6 \pm 3.3 \%$ estimated body fat) performed a circuit of three SE, with 3 sets of 6 repetitions, controlled by metronome, with a load of $85 \%$ (1-RM) and 3 minutes rest. The VDs of the internal saphenous vein (ISV), in right and left LL, were measured at baseline and immediately after each set with color eco-Doppler. Data were analyzed by means of a one-way ANOVA for repeated measures. There was a significant effect of time for the right and left VSI $(p=0.001)$, the VDs at baseline were higher than in series for both. Furthermore, between sets, there was a significant decrease between the first and second $(p=0.009)$ and an increase between the second and third series ( $p=0.027)$ for the right ISV. For the left ISV, only measured after the first and second are different $(p=0.001)$. There was a trend toward reduction in VD after the acute bout of strength exercise, indicating that their practice is efficient for the performance of this structure.

Keywords: varicose veins, adults, lower extremity, resistance training, athletic performance

Artigo recebido a 16.10.2013; $1^{\text {a }}$ Revisão 29.11.2013; 2 ${ }^{\text {a }}$ Revisão 23.01.2014; Aceite 07.02.2014

${ }^{1}$ Instituto Federal de Educação Ciência e Tecnologia da Paraíba, Brasil

${ }^{2}$ Unipê - Centro Universitário de João Pessoa, Paraíba, Brasil

${ }^{3}$ Universidade Federal da Paraíba, Centro de Ciências da Saúde - Campus I, Paraíba, Brasil

${ }^{4}$ Faculdade Integrada de Patos - Paraíba, Brasil

${ }^{5}$ Universidade Federal do Rio de Janeiro, Rio de Janeiro, Brasil

${ }^{6}$ Universidade de Trás-os-Montes e Alto Douro; Centro de Investigação em Desporto, Saúde e Desenvolvimento Humano, Vila Real, Portugal

* Autor correspondente: Universidade de Trás-os-Montes e Alto Douro, Parque Desportivo da UTAD, 5001-801 Vila Real - Portugal; E-mail: josevilaca@utad.pt 


\section{INTRODUÇÃO}

Os efeitos cardioprotetores do exercício físico têm sido referidos pelas principais associações médicas internacionais (ACSM, 2009; Garber et al., 2011; WHO, 2010) tanto para indivíduos saudáveis como para aqueles que possuem doença ou fatores de risco cardiovascular. Contudo, a análise dos efeitos do exercício físico nas doenças do sistema cardiovascular é efetuada com exercícios predominantemente aeróbios (Sophromadze \& Chabashvili, 2006) e com amostras de idade avançada e diagnóstico positivo de doenças venosas (Ciuffetti, Lombardini, Pasqualini, Vaudo, \& Lupattelli, 1999; Ibegbuna, Delis, Nicolaides, \& Aina, 2003; Klonizakis, Tew, Michaels, \& Saxton, 2009).

Neste contexto, o tipo de exercício recomendado pela literatura, para indivíduos com doenças venosas, é o aeróbio (Kahn et al., 2003), pelo fato deste ser capaz de melhorar o mecanismo de queda da pressão venosa e aumentar o fluxo venoso. A contração muscular funciona como um efeito de bomba muscular, proporcionando uma pressão de perfusão (Shiotani et al., 2002), o que auxilia o retorno sanguíneo dos membros inferiores até o coração, visto que, neste caminho a força da gravidade tem que ser superada (Padberg, Johnston, \& Sisto, 2004).

Em relação ao Treino de Força (TF), a utilização de exercícios que solicitem os grupos musculares dos membros inferiores, principalmente os músculos da face posterior da perna, tem-se demonstrado como uma atividade capaz de diminuir o refluxo sanguíneo, aprimorar a competência das veias e, consequentemente, promover redução dos desconfortos causados por disfunção das estruturas venosas (Padberg et al., 2004). No entanto poucas são as evidências científicas sobre o efeito da aplicação de exercícios do treino de força nas estruturas venosas, tornando-se difícil encontrar dados capazes de corroborar ideias, e existindo lacunas no conhecimento e respostas não conclusivas (Kumar, Seward, Wilcox, \& Torella, 2010; Padberg et al., 2004).
Deste modo, o objetivo do presente estudo é observar o efeito agudo de exercícios de treino de força sobre o sistema venoso superficial dos membros inferiores (MI) em homens treinados, por meio da mensuração do diâmetro venoso (DV). Esta pesquisa tem como hipótese principal que o efeito imediato destes exercícios não compromete o diâmetro venoso, e sim, possibilita a melhoria funcional deste sistema. Ademais, a opção pela inserção de indivíduos do sexo masculino é devido ao sexo feminino representar um fator de risco para doenças venosas (Beebe-Dimmer, Pfeifer, Engle, \& Schottenfeld, 2005; Seidel, Miranda Jr., Juliano, \& Novo, 2005).

\section{MÉTODO}

\section{Participantes}

A amostra foi composta por 22 homens, praticantes de treino de força, com uma média de $22 \pm 2$ anos de idade, $81.2 \pm 13.4 \mathrm{~kg}$ de massa corporal, $173.0 \pm 4.0 \mathrm{~cm}$ de estatura e $18.6 \pm 3.3 \%$ de percentagem de gordura estimada.

O cálculo amostral foi estimado por meio do programa estatístico GPower, adotou-se o erro de estimação $\alpha$ de $5 \%$, estimativa do tamanho do efeito de 0.5 , o poder $\beta$ de $80 \%$, e nível de confiança de $95 \%$, o que resultou no número mínimo de 17 indivíduos.

Os critérios de inclusão foram: (i) praticassem exercícios de força há pelo menos, três meses, com uma frequência semanal de 3 vezes; (ii) não fumassem; (iii) não ingerissem hormônios de qualquer natureza; (iv) não apresentassem sinais e/ou sintomas de doenças venosas periféricas; (v) não tivessem realizado nenhum procedimento cirúrgico venoso; $\mathrm{e}$ (vi) não apresentassem índice de massa corporal (IMC) superior a $28 \mathrm{~kg} / \mathrm{m}^{2}$. Como critério de exclusão: não conseguir executar os exercícios de TF durante a sessão experimental ou não comparecer ao dia de coleta de dados. Este estudo seguiu todos os procedimentos estabelecidos na declaração de Helsínquia sobre a ética na pesquisa em humanos. 


\section{Instrumentos e Procedimentos}

\section{Medidas antropométricas}

Para a caracterização antropométrica da amostra foi usado para medir a massa corporal uma balança antropométrica (Filizola, São Paulo, Brasil) e a estatura um estadiómetro (Cardiomed, Curitiba, Brasil). Os procedimentos para obtenção destas medidas decorreram de acordo com Eston, Eston e Reilly (2009). Para avaliar a percentagem de gordura estimada foi utilizado um aparelho de bioimpedância tetrapolar Bodystat 1500 (Bodystat, Ilha de Men, Reino Unido), utilizado como indica Lukaski, Bolonchuk, Hall e Siders (1986).

\section{Determinação da carga máxima (1-RM)}

A determinação da carga máxima (1-RM) foi efetuada quatro dias antes da coleta de dados, com intuito de minimizar o efeito residual de uma sessão de exercício resistido sobre a sessão experimental (Miles et al., 2008). Os indivíduos realizaram testes de repetições múltiplas nos exercícios de agachamento, avanço e flexão plantar em pé, com valor de 1-RM determinado segundo o modelo matemático de estimação de carga máxima proposto por Brzycki (1993). Previamente ao teste, cada sujeito realizou um aquecimento geral, por cinco minutos, numa bicicleta ergométrica (Monark, Suécia), a 25W e a $60 \mathrm{rpm}$. Na sequência, realizou-se um aquecimento específico com seis a dez repetições com $50 \%$ de carga a ser utilizada no teste, estipulada pelo próprio sujeito, dada a sua experiência prévia com os exercícios de TF. Após repouso de três minutos o teste foi iniciado. Para determinação do 1-RM, os sujeitos foram orientados a estimar uma carga para que tentassem executar no máximo de dez repetições sem haver falha mecânica. Ajustes na carga, com intervalos de três minutos, eram realizados quando o número de repetições excedia o valor estabelecido, ou era menos que sete. Este intervalo de repetições parece ser o mais adequado para estimar os valores de 1-RM (Whisenant, Panton, East, \& Broeder, 2003). Os escores médios, com o intervalo de confiança de $95 \%$, em relação à carga $(\mathrm{kg})$ e número de repetições para o agachamento foi de $71.27 \pm 0.78(69.63-72.91) \mathrm{kg}$ e $8.13 \pm 0.18$ (7.74-8.53) repetições; para o avanço foi de $50.63 \pm 0.64(49.28-51.98) \mathrm{kg}$ e $8.72 \pm 0.19$ (8.31-9.14) repetições; e, para a flexão plantar em pé foi de $82.27 \pm 0.78$ (80.63$83.91) \mathrm{kg}$ e $9.18 \pm 0.19$ (8.77-9.58) repetições. A cadência foi determinada por metrónomo (Timex, North Little Rock, EUA) em compasso binário (2/2) e 50 bpm.

Mensuração dos diâmetros venosos e refluxo venoso Os diâmetros venosos foram medidos por um médico angiologista. A medida ocorreu durante a sessão experimental, em horários (07h00min as 08h30min) e dias padronizados. Utilizou-se um aparelho de eco-Doppler colorido (Shimatzu, Kyoto, Japão), com transdutor linear multifrequencial de $5-10 \mathrm{MHz}$. O diâmetro venoso (DV) foi determinado em sete porções anatómicas, sendo quatro pontos no segmento da coxa e três na perna, na veia safena interna (VSI) do membro direito e esquerdo, no repouso e imediatamente após as séries de treino de força, totalizando 56 medidas por indivíduo (16 na coxa e 12 na perna, em cada membro). Os locais de medição foram determinados conforme Seidel et al. (2005). O refluxo venoso foi analisado sempre durante a mensuração dos DV, com os indivíduos na posição de pé, usando a manobra de Valsalva e compressão e descompressão bruscas distais para detetar a presença ou não de refluxo, sendo considerado como refluxo venoso o tempo maior que 0.5 segundos de movimento retrógrado do fluxo venoso na veia (Pavei et al., 2008; Quigley, Raptis, \& Cashman, 1994; Souza et al., 2005).

As sessões ocorreram numa sala ampla e bem iluminada com $20 \mathrm{~m}^{2}$, com temperatura de $22 \pm 2^{\circ} \mathrm{C}$ (Beurer, Ulm, Alemanha). Após 10 minutos de repouso na posição sentada, cada sujeito da amostra realizou a primeira medida (na posição de pé) do DV, da VSI, de ambos os membros inferiores. Posteriormente e após um aquecimento específico conforme indicam Gourgoulis, Aggeloussis, Kasimatis, Mavroma- 
tis e Garas (2003), seguiu-se a realização de três séries de um circuito de três exercícios de força, iniciando com o avanço, agachamento e por último, flexão plantar. Com o DV foi medido após a execução de cada série, sendo três medidas. Totalizando quatro medidas repetidas: uma inicial e três ao término de cada série de exercício de força para os MI.

As séries de força foram realizadas com intensidade de $85 \%$ de 1-RM, com pesos livres e barras (Panatta, Apiro, Itália) de diferentes quilagens. Foram desempenhadas três séries de 6 repetições e intervalos de 3 minutos entre as mesmas, com aquecimento de 10 minutos, perfazendo um tempo total de 30 a 50 minutos por sessão, conforme indicado pelo colégio americano de medicina esportiva (Garber et al., 2011), para este tipo de exercício. A execução de cada exercício foi ritmada por metrónomo, ajustado em $50 \mathrm{bpm}$, com compasso binário $(2 / 2)$, de forma a assegurar que todos os sujeitos empregassem o mesmo tempo para cada repetição. Para verificar a intensidade da sessão, empregou-se a escala de Perceção Subjetiva de Esforço OMNI-RES, adaptada para exercícios de força. O pesquisador principal do estudo orientou as sessões, monitorou a intensidade e o volume, e corrigiu a postura dos participantes durante a prática dos exercícios.

\section{Análise Estatística}

Os dados foram tabulados e analisados no pacote estatístico SPSS (Statistical Package for
Social Sciences), versão 16.0 para Windows. A estatística descritiva foi composta pela determinação dos valores de média e desvio-padrão (DP), além dos valores máximo e mínimo para as características antropométricas. Antes da análise inferencial, a esfericidade dos dados foi verificada por meio do teste de Mauchly, sendo que, ocorreu violação deste pressuposto nas medidas do membro direito e esquerdo, desta forma, a correção de Greenhouse-Geisser foi utilizada para modificar os graus de liberdade. Em seguida, foi analisado, por meio de ANOVA de medidas repetidas, com o modelo 1 grupo $\times 4$ momentos para as medidas dos diâmetros venosos durante a sessão de exercício de força. Foi efetuada a estimativa do tamanho do efeito através do valor do eta quadrado parcial $\left(\eta_{\mathrm{p}}^{2}\right)$, segundo Cohen (1988). O nível de significância foi estabelecido em 5\%.

\section{RESULTADOS}

A Tabela 1 demonstra a resposta dos diâmetros venosos da veia safena interna do membro direito (VSIdir), antes e imediatamente após a execução das três séries de exercícios de TF. Os valores desta variável tenderam a diminuir após a primeira série de exercício. Contudo, não foi detetado refluxo venoso em nenhum ponto durante o exercício.

Em relação aos diâmetros venosos do membro esquerdo (VSIesq), verificou-se, descritivamente, que na maioria dos pontos mensurados as médias tenderam a diminuir,

Tabela 1

Diâmetro venoso da VSI do membro inferior direito durante a sessão de exercício de força em homens treinados $(n=22)$

\begin{tabular}{ccccc}
\hline \multirow{2}{*}{ Ponto anatómico } & \multicolumn{4}{c}{ Diâmetro venoso $(\mathrm{mm})(\mathrm{M} \pm \mathrm{DP})$} \\
\cline { 2 - 5 } & VSI Repouso & VSI após $1^{\mathrm{a}}$ série & VSI após 2 ${ }^{\mathrm{a}}$ série & VSI após 3 $^{\mathrm{a}}$ série \\
\hline 1- Coxa & $3.55 \pm 0.92$ & $3.28 \pm 0.88$ & $2.99 \pm 0.72$ & $3.09 \pm 0.68$ \\
2- Coxa & $3.38 \pm 0.89$ & $3.04 \pm 0.75$ & $2.85 \pm 0.71$ & $2.95 \pm 0.71$ \\
3- Coxa & $3.21 \pm 0.87$ & $2.80 \pm 0.65$ & $2.81 \pm 0.73$ & $2.84 \pm 0.75$ \\
4- Coxa & $3.15 \pm 1.08$ & $2.71 \pm 0.99$ & $2.76 \pm 0.93$ & $2.73 \pm 0.82$ \\
5- Perna & $2.64 \pm 0.55$ & $2.30 \pm 0.60$ & $2.24 \pm 0.46$ & $2.30 \pm 0.42$ \\
6- Perna & $2.53 \pm 0.41$ & $2.30 \pm 0.49$ & $2.20 \pm 0.47$ & $2.32 \pm 0.41$ \\
7- Perna & $2.45 \pm 0.40$ & $2.27 \pm 0.50$ & $2.23 \pm 0.48$ & $2.27 \pm 0.41$ \\
\hline
\end{tabular}


Tabela 2

Diâmetro venoso $(\mathrm{mm})$ da VSI do membro inferior esquerdo durante a sessão de exercício de força em homens treinados $(n=22)$

\begin{tabular}{ccccc}
\hline \multirow{2}{*}{ Ponto anatómico } & \multicolumn{4}{c}{ Diâmetro venoso $(\mathrm{mm})(\mathrm{M} \pm \mathrm{DP})$} \\
\cline { 2 - 5 } & VSI Repouso & VSI após $1^{\mathrm{a}}$ série & VSI após 2 ${ }^{\mathrm{a}}$ série & VSI após 3 $^{\mathrm{a}}$ série \\
\hline 1- Coxa & $3.68 \pm 1.01$ & $3.40 \pm 0.90$ & $3.34 \pm 0.89$ & $3.38 \pm 0.76$ \\
2- Coxa & $3.50 \pm 0.95$ & $3.21 \pm 0.82$ & $3.30 \pm 0.83$ & $3.27 \pm 0.76$ \\
3- Coxa & $3.52 \pm 0.97$ & $3.08 \pm 0.84$ & $3.26 \pm 0.79$ & $3.23 \pm 0.73$ \\
4- Coxa & $3.32 \pm 1.10$ & $2.95 \pm 1.03$ & $3.16 \pm 1.03$ & $3.02 \pm 0.79$ \\
5- Perna & $2.88 \pm 0.83$ & $2.61 \pm 0.85$ & $2.80 \pm 0.77$ & $2.65 \pm 0.51$ \\
6- Perna & $2.65 \pm 0.52$ & $2.34 \pm 0.44$ & $2.57 \pm 0.48$ & $2.47 \pm 0.47$ \\
7- Perna & $2.60 \pm 0.49$ & $2.47 \pm 0.51$ & $2.52 \pm 0.44$ & $2.47 \pm 0.42$ \\
\hline
\end{tabular}

Nota: VSI - Veia safena interna

significativamente $(p<0.05)$ após o início do exercício (Tabela 2). Nesta veia também não houve a ocorrência de refluxo venoso durante a sessão experimental.

Quanto ao efeito do exercício de força sobre a estrutura venosa, verifica-se que, para o membro direito, houve um efeito significativo $\left(F_{(2.38,364.71)}=52.84, p=0.001, \eta_{\mathrm{p}}{ }^{2}=0.25\right)$, com elevado tamanho do efeito. As medidas do repouso foram maiores do que as medidas após a primeira série $(p=0.001)$, após a segunda $(p=0.001)$ e após a terceira série $(p=0.001)$. Em adição, refere-se a diminuição significativa entre as medidas após a primeira e segunda séries $(p=0.009)$ e o aumento entre a segunda e terceira séries $(p=0.027)$ (Figura 1$)$. Em relação à VSIesq, verificou-se efeito significativo do tempo $\left(F_{(2.72,416.56)}=22.69, p=0.001\right.$, $\left.\eta_{\mathrm{p}}{ }^{2}=0.13\right)$, porém com baixo tamanho do efeito. Nestas veias também houve uma queda significativa do repouso para primeira $(p=$ $0.001)$, segunda $(p=0.001)$ e terceira $(p=$ 0.001 ) séries, ademais observa-se uma diferença entre das medidas após a primeira e segunda série $(p=0.001)$, momento em que houve um aumento do DV (Figura 1).

Quanto à perceção subjetiva de esforço (PSE), durante as séries de exercício de força, em relação aos músculos utilizados, observa-se um efeito significativo do tempo $\left(F_{(2,42)}=\right.$ 101.67, $p=0.001, \eta_{\mathrm{p}}^{2}=0.82$ ), com elevado tamanho de efeito. A PSE aumentou ao longo da sessão experimental, sendo que a medida após a primeira série foi de $6.77 \pm 0.81$, classificada como atividade pouco difícil. Após a segunda série de $8.05 \pm 0.84$ categorizando o exercício como difícil, e após a execução da última série foi de $9.23 \pm 0.61$, valor considerado como "extremamente difícil" para a perceção do exercício de força.

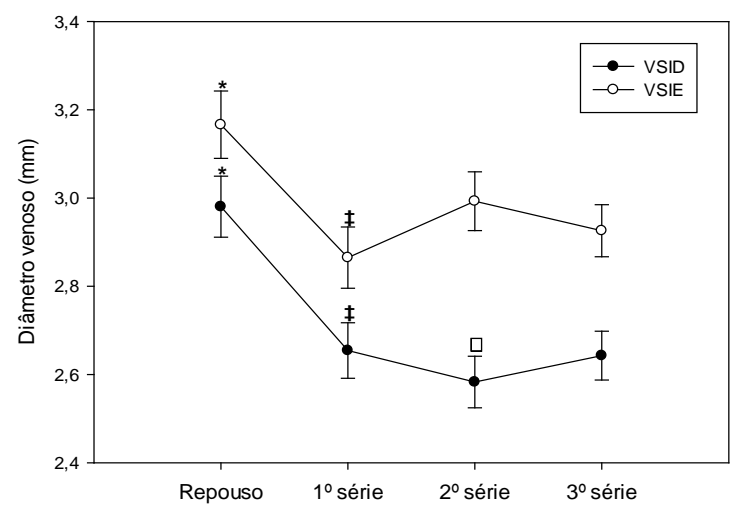

Figura 1. Medidas do diâmetro venoso (mm) da veia safena interna do membro inferior direito (VSID) e esquerdo (VSIE), nas distintas situações durante a sessão de exercício de força em homens treinados $(\mathrm{n}=22) .{ }^{*}$ Diferença do repouso para as três séries $(p<0.05)$; $\ddagger$ diferença entre a $1^{\mathrm{a}}$ e $2^{\mathrm{a}}$ série $(p<0.05)$;

$\theta$ diferença entre a $2^{\mathrm{a}}$ e $3^{\mathrm{a}}$ série $(p<0.05)$

\section{DISCUSSÃO}

Os resultados deste estudo parecem revelar que uma sessão de exercício de força, para os 
membros inferiores, não afeta de forma negativa a função das estruturas venosas superficiais, deste segmento. A afirmação anterior é suportada pela não observação de refluxo venoso, em nenhum momento da sessão experimental, demonstrando, que o efeito agudo da prática, deste tipo de atividade, não causa prejuízo ao sistema venoso superficial do membro inferior (SVSMI). Em adição, pode-se referir, o fato das VSI tenderem a diminuir da situação do repouso para as medidas ao longo das séries de exercícios de TF. Acrescenta-se, que em relação ao exercício aeróbio (corrida), Sophromadze e Chabashvili (2006) não observaram um efeito prejudicial, nas veias dos membros inferiores, ao nível da complacência e da eficiência das suas válvulas, em atletas de futebol e wrestlers de alto nível. Outro aspeto, que merece ser referido, é que após cessar o exercício, as veias dos jogadores de futebol tiveram um retorno à condição inicial mais rápido do que o grupo de wrestlers. Contudo, o observado, pode ter resultado das diferenças iniciais, significativas, no diâmetro venoso e no fluxo sanguíneo, e superiores nos sujeitos jogadores de futebol em relação aos wrestlers. Os autores atribuem essas diferenças às possíveis adaptações fisiológicas da circulação ascendente, que é facilitada pelo tipo de contração muscular dos MI na prática do futebol (Sophromadze \& Chabashvili, 2006).

Os diâmetros venosos estão fortemente relacionados com as insuficiências do sistema venoso periférico, como a insuficiência venosa crónica (IVC). A dilatação de um vaso sanguíneo pode provocar o refluxo venoso e, consequentemente, um aumento na pressão venosa local, especialmente nos membros inferiores (Eifell, Ashour, \& Lees, 2006), onde devido à ação da força da gravidade, necessita de maior bombeamento para o seu trajeto ascendente ao átrio direito (Padberg et al., 2004).

Sabe-se que o exercício aeróbio (Ciuffetti et al., 1999; Ibegbuna et al., 2003; Klonizakis et al., 2009) e com sobrecarga muscular (Silva et al., 2010) promove uma melhoria na função venosa dos membros inferiores em pacientes com IVC. Esta melhoria já foi observada em pacientes com trombose venosa profunda com o exercício de caminhada em tapete rolante e, igualmente, observado uma diminuição, significativa, do volume do membro afetado (Kahn et al., 2003). O IVC é uma patologia multifatorial, contudo a exposição prolongada do vaso a elevadas pressões venosas parece ser um dos aspetos mais importantes no processo de desenvolvimento da doença (Eifell et al., 2006). O efeito hipotensivo após exercício aeróbio (Casonatto \& Polito, 2009) e de treino de força (Rodrigues-da-Silva, Lima, Rodrigues, Júnior, \& Ritti-Dias, 2013) pode ter neste aspeto um papel importante, podendo ser uma das razões dos resultados observados no presente estudo.

As medidas dos diâmetros venosos apresentaram-se reduzidas após o desempenho das séries de exercícios de treino de força. Apesar destas alterações, as veias demonstraram estar adequadas quanto à sua função e competência, uma vez que, após o exame, o médico não referiu à presença de refluxo sanguíneo na amostra investigada quando estas estruturas são submetidas à sobrecarga tensional dos músculos, sugerindo a suficiência do sistema valvular destas veias, que se localizam acima da musculatura da perna. Em indivíduos saudáveis e com idade avançada (60 \pm 4 anos) foi observada uma rápida queda da pressão venosa do tornozelo e aumento do fluxo venoso da perna em resposta aguda ao exercício em cicloergómetro (Shiotani et al., 2002). Este fato deve-se a durante o exercício, a contração muscular aumenta a pressão fora das veias e impulsiona de volta o sangue ao coração, reduzindo o gradiente de pressão hidrostática necessário para a formação de edema (Stick, Grau, \& Witzleb, 1989).

Engelhorn et al. (1997) evidenciaram que o diâmetro da veia safena é um forte indicador da insuficiência valvular venosa e que, por meio da análise isolada, desta única medida (diâmetro), é possível predizer a existência de um refluxo com um nível de exatidão de $70 \%$. Além disso, a quantificação do diâmetro apre- 
senta relação direta com o refluxo venoso (Mdez-Herrero et al., 2007). É pertinente ressaltar que o equipamento empregue para as medições dos DVs, é adotado como padrão para o diagnóstico e demonstração da anatomia venosa dos membros inferiores, garantido assim a validade e a fiabilidade dos dados obtidos (Engelhorn et al., 1997; Seidel et al., 2005).

De acordo com dados apresentados por van Duijnhoven et al. (2008) que, ao aplicarem um programa de treino de força, com electroestimulação, não identificaram aumento no diâmetro da veia poplítea; no entanto, este estudo avaliou uma espécie de treino vibratório com aproximadamente $19 \mathrm{~Hz}$, e com execução de exercícios de força estáticos, portanto de baixa intensidade e isométricos. Em referência às investigações que objetivaram analisar os efeitos do exercício de força sobre a competência vascular, especialmente, no que concerne ao treinamento dos músculos gastrocnémio e sóleo, os resultados indicam que o fortalecimento desses grupos musculares promove alterações benéficas na hemodinâmica venosa. Tais como, a diminuição do refluxo sanguíneo, redução do volume venoso funcional e da fração de volume residual, além do aumento da fração de ejeção sanguínea. Em adição, foi observado num programa de exercício, o qual incluiu treino de força juntamente com meias de compressão, uma diminuição do refluxo venoso em pacientes com doença venosa crónica (Zajkowski, Draper, Bloom, Henke, \& Wakefield, 2006).

Quanto à corroboração ou contestação desses dados, com os resultados de outros estudos, evidencia-se que os mecanismos fisiológicos capazes de explicar possíveis alterações ainda não estão amplamente esclarecidos. Em relação à redução dos DVs, após o início dos exercícios, verifica-se uma vasoconstrição dos vasos em resposta a sobrecarga muscular atribuída pelas atividades físicas, que pode ser favorecida pelo efeito de sucção venosa dos músculos inspiratórios (Miller, Pegelow, Jacques, \& Dempsey, 2005), e pela transferência de sangue para a circulação central devido à contração muscular e retorno impedido pelas válvulas venosas (Stick, Jaeger, \& Witzleb, 1992).

Durante o exercício físico há uma elevação da atividade do sistema simpático, que é um importante sistema vasopressor no controle da capacitância venosa (Pang, 2001). As contrações rítmicas da musculatura esquelética transferem uma considerável quantidade de energia cinética para o sangue venoso facilitando, assim, o retorno venoso ao coração (Miller et al., 2005). Nesse sentido, o exercício físico atua, igualmente, favorecendo o retorno venoso por meio da bomba muscular periférica da perna (González-Alonso et al., 2008). Entretanto, pesquisas enfatizam a investigação dessas alterações em relação ao exercício aeróbio (Padberg et al., 2004) e apontam a necessidade de respostas mais conclusivas, relacionadas às modificações venosas durante o exercício de força.

Como limitações deste estudo, citam-se a falta de medição da velocidade do fluxo venoso durante a sessão aguda de força, a não mensuração da pressão das veias ao longo do desempenho das séries, bem como a falta de controlo do padrão da respiração, visto que existem diferenças no retorno venoso, conforme o tipo de respiração adotado, diafragmática ou torácica (Miller et al., 2005). Neste estudo, o padrão respiratório foi contínuo, com tendência para o bloqueio durante a execução das séries, devido ao alto percentual de carga aplicado. Além disto, não se identificou o tempo necessário para que os diâmetros das veias retornem aos níveis iniciais.

Quanto às implicações práticas, destaca-se o fato desta pesquisa indicar que o desempenho de exercícios de força não comprometeu o funcionamento das veias superficiais do membro inferior, veias que estão relacionadas ao surgimento de varizes (Beebe-Dimmer et al., 2005), o que é um desfecho evitado devido à má aparência estética das veias dilatadas. Indicando aos profissionais que atuam com este tipo de exercícios em academias, clubes, ginásios ou 
ambientes públicos, que a prática de exercícios de treino de força, como o agachamento, flexão plantar e avanço, não causam danos às veias do sistema venoso superficial dos membros inferiores. Porém, ressalta-se que foram avaliados homens treinados. Não obstante, o treino de força também não promoveu alterações negativas nas veias superficiais do membro inferior em mulheres com doença venosa (Silva et al., 2010). Enfim, estes dados corroboram a ideia de que deve-se prescrever os exercícios de TF como formar de auxiliar no funcionamento do sistema venoso superficial dos membros inferiores (SVSMI).

Considera-se que o presente estudo, poderá servir como um parâmetro para direcionamento de pesquisas que investiguem o efeito do exercício de força para mulheres. A observação do efeito deste tipo de exercício, nesta população, torna-se pertinente, visto que o SVSMI dos indivíduos do sexo feminino apresenta-se mais frágil que os congéneres masculinos, principalmente devido a fatores hormonais. Assim, sugerem-se estudos futuros com populações do sexo feminino, sob condição de controlo de exposição de riscos.

\section{CONCLUSÕES}

A partir do desempenho agudo dos diâmetros venosos, sugere-se que a prática do exercício de força não promove alterações negativas na estrutura das veias de homens treinados. Adiciona-se que não houve refluxo venoso nos membros inferiores investigados, em que a tendência do diâmetro venoso é reduzir-se após a sessão aguda de exercício de força, indicando que a sua prática é eficiente para o desempenho desta estrutura, por não comprometê-la.

\footnotetext{
Agradecimentos:

Ao médico angiologista, Dr. Rodolfo Augusto Athayde Neto, do Departamento de Cirurgia da Universidade Federal da Paraíba, pela colaboração na realização dos exames.
}

\section{Conflito de Interesses:}

Nada a declarar.

\section{Financiamento:}

Nada a declarar.

\section{REFERÊNCIAS}

ACSM (2009). Progression Models in Resistance Training for Healthy Adults. Medicine \& Science in Sports \& Exercise, 41(3), 687-708. doi: 10.1249/MSS.0b013e3181915670

Beebe-Dimmer, J. L., Pfeifer, J. R., Engle, J. S., \& Schottenfeld, D. (2005). The epidemiology of chronic venous insufficiency and varicose veins. Annals of Epidemiology, 15(3), 175-184. doi: 10.1016/j.annepidem.2004.05.015

Brzycki, M. (1993). Strength testing - predicting a one-rep max from reps-to-fatigue. Journal of Physical Education, Recreation and Dance, 64(1), 88-90. doi: 10.1080/07303084.1993.10606684

Casonatto, J., \& Polito, M. D. (2009). Hipotensão pós-exercício aeróbio: Uma revisão sistemática. Revista Brasileira de Medicina do Esporte, 15, 151157. doi: 10.1590/S1517-86922009000200014

Ciuffetti, G., Lombardini, R., Pasqualini, L., Vaudo, G., \& Lupattelli, G. (1999). Circulating leucocyte adhesion molecules in chronic venous insufficiency. European Journal of Vascular Medicine, 28, 156-159. doi: 10.1024/0301-1526.28.3.156

Cohen, J. (1988). Statistical power analysis for the behavioral sciences ( $2^{\mathrm{a}}$ ed.). Hillsdale: Routledge.

Eifell, R. K., Ashour, H. Y., \& Lees, T. A. (2006). Comparison of new continuous measurements of ambulatory venous pressure (AVP) with conventional tiptoe exercise ambulatory AVP in relation to the CEAP clinical classification of chronic venous disease. Journal of Vascular Surgery, 44(4), 794-802. doi: 10.1016/j.jvs.2006. 06.007

Engelhorn, C., Engelhorn, A., Salles-Cunha, S., Picheth, F., Castro Jr, N., \& Dabul Jr, N. (1997). Relationship between reflux and greater saphenous vein diameter. Journal of Vascular Technology, 21 (3), 167-171.

Eston, R., Eston, R. G., \& Reilly, T. (2009). Kinanthropometry and Exercise Physiology Laboratory Manual: Anthropometry. Oxon: Routledge. 
Garber, C. E., Blissmer, B., Deschenes, M. R., Franklin, B. A., Lamonte, M. J., \& Lee, I.-M. (2011). Quantity and Quality of Exercise for Developing and Maintaining Cardiorespiratory, Musculoskeletal, and Neuromotor Fitness in Apparently Healthy Adults: Guidance for Prescribing Exercise. Medicine \& Science in Sports \& Exercise, 43(7), 1334-1359. doi: 1310.1249/MSS.1330b1 $013 \mathrm{e} 318213 \mathrm{fefb}$.

González-Alonso, J., Mortensen, S. P., Jeppesen, T. D., Ali, L., Barker, H., \& Damsgaard, R. (2008). Haemodynamic responses to exercise, ATP infusion and thigh compression in humans: Insight into the role of muscle mechanisms on cardiovascular function. The Journal of Physiology, 586(9), 2405-2417. doi: 10.1113/ jphysiol.2008.152058

Gourgoulis, V., Aggeloussis, N., Kasimatis, P., Mavromatis, G., \& Garas, A. (2003). Effect of a submaximal half-squats warm-up program on vertical jumping ability. The Journal of Strength \& Conditioning Research, 17(2), 342-344.

Ibegbuna, V., Delis, K. T., Nicolaides, A. N., \& Aina, O. (2003). Effect of elastic compression stockings on venous hemodynamics during walking. Journal of Vascular Surgery, 37(2), 420-425. doi: 10.1067/mva.2003.104S0741521402752452

Kahn, S. R., Azoulay, L., Hirsch, A., Haber, M., Strulovitch, C., \& Shrier, I. (2003). Acute effects of exercise in patients with previous deep venous thrombosis: Impact of the postthrombotic syndrome. Chest, 123(2), 399-405.

Klonizakis, M., Tew, G., Michaels, J., \& Saxton, J. (2009). Exercise training improves cutaneous microvascular endothelial function in postsurgical varicose vein patients. Microvascular Research, 78(1), 67-70. doi: 10.1016/j.mvr.2009. 03.002

Kumar, S., Seward, J., Wilcox, A., \& Torella, F. (2010). Influence of muscle training on resting blood flow and forearm vessel diameter in patients with chronic renal failure. British Journal of Surgery, 97(6), 835-838. doi: 10.1002/bjs. 7004

Lukaski, H. C., Bolonchuk, W. W., Hall, C. B., \& Siders, W. A. (1986). Validation of tetrapolar bioelectrical impedance method to assess human body composition. Journal of Applied Physiology, 60(4), 1327-1332.

Mdez-Herrero, A., Gutierrez, J., Camblor, L., Carreno, J., Llaneza, J., \& Rguez-Olay, J. (2007). The relation among the diameter of the great sa- phenous vein, clinical state and haemodynamic pattern of the saphenofemoral junction in chronic superficial venous insufficiency. Phlebology, 22(5), 207-213.

Miles, M. P., Andring, J. M., Pearson, S. D., Gordon, L. K., Kasper, C., \& Depner, C. M. (2008). Diurnal variation, response to eccentric exercise, and association of inflammatory mediators with muscle damage variables. Journal of Applied Physiology, 104(2), 451-458. doi: 10.1152/jappl physiol.00572.2007

Miller, J. D., Pegelow, D. F., Jacques, A. J., \& Dempsey, J. A. (2005). Effects of augmented respiratory muscle pressure production on locomotor limb venous return during calf contraction exercise. Journal of Applied Physiology, 99(5), 18021815. doi: 10.1152/japplphysiol.00278.2005

Padberg, F. T., Johnston, M. V., \& Sisto, S. A. (2004). Structured exercise improves calf muscle pump function in chronic venous insufficiency: A randomized trial. Journal of Vascular Surgery 39(1), 79-87. doi: 10.1016/j.jvs.2003. 09.036

Pang, C. C. (2001). Autonomic control of the venous system in health and disease: Effects of drugs. Pharmacology \& Therapeutics, 90(2-3), 179230. doi: 10.1016/S0163-7258(01)00138-3

Pavei, P., Vecchiato, M., Spreafico, G., Giraldi, E., Ferrini, M., \& Baccaglini, U. (2008). Natural history of recurrent varices undergoing reintervention: A retrospective study. Dermatologic Surgery, 34, 1676-1682. doi: 10.1111/j.15244725. 2008.34345.x

Quigley, F. G., Raptis, S., \& Cashman, M. (1994). Duplex ultrasonography of recurrent varicose veins. Cardiovascular Surgery, 2(6), 775-777.

Rodrigues-da-Silva, A. J., Lima, A. A., Rodrigues, S. C., Júnior, C. C., \& Ritti-Dias, R. M. (2013). Efeito da fadiga induzida pelo treino de força na resposta da pressão arterial em sujeitos hipertensos: Uma revisão sistemática. Motricidade, 9, 23-30. doi: 10.6063/motricidade.9(1). 2460

Seidel, A., Miranda Jr., F., Juliano, Y., \& Novo, N. (2005). Relationship between the diameter of great saphenous vein and body mass index. Jornal Vascular Brasileiro, 4, 265-269. doi: 10.1590/ S1677-54492005000300008

Shiotani, I., Sato, H., Yokoyama, H., Ohnishi, Y., Hishida, E., \& Kinjo, K. (2002). Muscle pumpdependent self-perfusion mechanism in legs in normal subjects and patients with heart failure. 
70 | A Araújo Júnior, RT Araújo, RR Aniceto, LS Oliveira, J Novaes, MSC Sousa, J Vilaça-Alves

Journal of Applied Physiology, 92(4), 1647-1654. doi: 10.1152/japplphysiol.01096.2000

Silva, G. C. C., Medeiros, R. J. D., Oliveira, L. S., Araújo Júnior, A. T., Aniceto, R. R., Sousa, M. S. C. (2010). Treinamento de sobrecarga muscular não afeta o diâmetro das principais veias dos membros inferiores em mulheres adultas com insuficiência venosa. Revista Brasileira de Medicina do Esporte, 16(6), 413-417. doi: 10.1590/S1517-86922010000600003

Sophromadze, Z., \& Chabashvili, N. (2006). Higher and lower extremity vein color duplex sonography in highly qualified football players and wrestlers. Georgian Medical News, 135, 106108.

Souza, G. G., Pereira, A. H., Costa, L. F. M., Gassen, E., Nhuch, C., \& Renosto, R. (2005). Teste de Valsalva ortostático no refluxo venoso. Jornal Vascular Brasileiro, 4, 270-274. doi: 10.1590/ S1677-54492005000300009

Stick, C., Grau, H., \& Witzleb, E. (1989). On the edema-preventing effect of the calf muscle pump. European Journal of Applied Physiology and Occupational Physiology, 59(1-2), 39-47. doi: 10.1007/BF02396578
Stick, C., Jaeger, H., \& Witzleb, E. (1992). Measurements of volume changes and venous pressure in the human lower leg during walking and running. Journal of Applied Physiology, 72 (6), 2063-2068.

van Duijnhoven, N. T., Bleeker, M. W., de Groot, P. C., Thijssen, D. H., Felsenberg, D., Rittweger, J., \& Hopman, M. T. (2008). The effect of bed rest and an exercise countermeasure on leg venous function. European Journal of Applied Physiology, 104(6), 991-998.

Whisenant, M. J., Panton, L. B., East, W. B., \& Broeder, C. E. (2003). Validation of submaximal prediction equations for the 1 repetition maximum bench press test on a group of collegiate football players. The Journal of Strength $\mathcal{E}$ Conditioning Research, 17(2), 221-227.

WHO (2010). Global recommendations on physical activity for health. Geneva: WHO.

Zajkowski, P. J., Draper, T., Bloom, J., Henke, P. K., \& Wakefield, T. W. (2006). Exercise with compression stockings improves reflux in patients with mild chronic venous insufficiency. Phlebology, 21(2), 100-104. doi: 10.1258/0268 35506777304764 quando especificado em contrário e nos conteúdos retirados de outras fontes bibliográficas. 